\title{
Effects of Deep Brain Stimulation of the Subthalamic Nucleus on the Postoperative Levodopa Response: One Year Follow Up
}

\author{
Ersoy KOCABICAK ${ }^{1,2, *}$, Onur YILDIZ1,3,*, Dursun AYGUN ${ }^{1,3}$, Yasin TEMEL $^{4}$ \\ ${ }^{1}$ Ondokuz Mayıs University, Health Practise and Research Hospital, Neuromodulation Center, Samsun, Turkey \\ ${ }^{2}$ Ondokuz Mayıs University, Faculty of Medicine, Department of Neurosurgery, Samsun, Turkey \\ ${ }^{3}$ Ondokuz Mayıs University, Faculty of Medicine, Department of Neurology, Samsun, Turkey \\ ${ }^{4}$ Maastricht University Medical Center, Department of Neurosurgery, Maastricht, Netherlands \\ ${ }^{*}$ These authors have contributed equally
}

This study has been presented at the International Congress of Parkinson's Disease \& Movement Disorders between 22 and 26 September 2019 at Nice, France

Corresponding author: Ersoy KOCABICAK ersoykocabicak@gmail.com

\section{ABSTRACT}

AIM: To investigate the effect of preoperative levodopa responsiveness to clinical outcomes in the first postoperative year, and to evaluate the changes in the postoperative levodopa responsiveness in patients undergoing subthalamic nucleus (STN) deep brain stimulation (DBS).

MATERIAL and METHODS: Forty-nine Parkinson's Disease (PD) patients undergoing bilateral DBS of the STN were included in this study. Their clinical motor symptoms were assessed preoperatively by UPDRS Part III score in both OFF and ON medication states. Postoperatively, the assessments were obtained in three consecutive conditions. Preoperatively and postoperatively, the percentage difference between these two scores was evaluated as levodopa response.

RESULTS: Mean age was $54.6 \pm 9$ years (27-70). Levodopa response significantly decreased postoperatively by $56 \%$ a year. Compared with preoperative med on and postoperative stim on / med on scores, the clinical results of the first year were obtained and an improvement of $25 \%$ on the UPDRS 3 score was observed. Compared with preoperative levodopa response and clinical outcomes, better clinical results were obtained in patients with higher preoperative levodopa response $(p<0.05)$.

CONCLUSION: In this study, we confirm that the response of L-dopa decreases after DBS of the STN. The reasons for this finding are not clear. However, DBS of the STN allows for the reduction of PD medications and improvement of daily life activities, motor function, motor fluctuations, and dyskinesia.

KEYWORDS: Parkinson's disease, Deep brain stimulation, Levodopa response, Subthalamic nucleus

ABBREVIATIONS: CT: Computed tomography, DBS: Deep brain stimulation, LEDD: Leva-Dopa daily dosage, MER: Microelectrode recording, MMT: Mini mental test, MR: Magnetic resonance, MRI: Magnetic resonance imaging, SNr: Substantia nigra pars reticulata, STN: Subthalamic nucleus, PD: Parkinson's disease, UPDRS: Unified Parkinson's disease rating scale

\section{INTRODUCTION}

$\mathrm{P}$ arkinson's disease (PD) results from dopaminergic deficiency and the primary treatment of PD is dopamine replacement therapy. Dopamine agonists and levodopa allows for controlling motor symptoms of PD for 5 to 10 years on average. However, motor fluctuations and dyskinesia are the main problems in the majority of patients in the moderateto-advanced stages of the disease $(23,24)$. Subthalamic nucleus (STN) deep brain stimulation (DBS) has been an
Ersoy KOCABICAK (1) : 0000-0003-3445-1734 Onur YILDIZ (i) : 0000-0002-2796-8770
Dursun AYGUN (D) : 0000-0002-7079-6142

Yasin TEMEL (1) : 0000-0002-3589-5604 
effective surgical method used in mid- to advanced-stage Parkinson's disease for nearly three decades. Despite the debate over its mechanisms of action, STN DBS, like the dopaminergic therapy, is suggested to relieve the symptoms of $\mathrm{PD}$ by regulating the rate of discharge of the basal ganglia output nucleus $(2,4,11)$. In parallel, STN DBS has gained wide recognition that the preoperative level of the levodopa responsiveness predicts the degree of response to STN-DBS in PD (20). An important criterion for the success of DBS is the selection of patients fit for this mode of treatment. A favorable response to levodopa; which is a preoperative reduction by no less than $25 \%$ in the UPDRS-III motor scores from an "off"medication state to "on"-medication, is still regarded as one of the most important criteria in determining the suitability of a patient for STN-DBS $(17,18,28)$. Nevertheless; in some recent publications, it has been shown that the degree of preoperative L-dopa response does not predict long-term outcomes of STN-DBS (20) with case reports indicating that some patients respond to STN-DBS even though there was not a previous response to L-dopa preoperatively (29). While being effective in PD, the effects of STN-DBS on the postoperative levodopa responsiveness have not been established clearly. It has been reported in some studies that there is a marked loss of levodopa response in the postoperative period; however, a clarified rationale has not been suggested $(15,21)$. Along with the clinical outcomes achieved in the first year after STN DBS; our study aimed to investigate the effects of the preoperative levodopa responsiveness on the clinical outcomes observed in the postoperative one-year and to evaluate the changes in the postoperative levodopa responsiveness.

\section{MATERIAL and METHODS}

The PD patients, who underwent STN-DBS from October 2011 to December 2015 in the DBS center of Ondokuz Mayıs University, were included in the study. The data were obtained from the patients at the end of a one-year followup. The clinical motor symptoms during the preoperative year before STN-DBS and those in the postoperative year were evaluated with the United Parkinson's Disease Rating Scale (UPDRS) Part III scores. Preoperatively and postoperatively, the "off"-medication state was examined at least 12 hours after the discontinuation of antidopaminergic treatments. The "on"-medication state was examined after administering a 1.5-fold dose of levodopa in the morning. Three consecutive examinations were postoperatively carried out depending on whether stimulation was on or off (Medication Off-Stimulation Off, Medication Off-Stimulation On, and Medication OnStimulation On). The percentage differences from the "off"state to the "on"-state in the UPDRS III scores between the preoperative and postoperative periods were calculated and accepted as the levodopa response under the influence of stimulation. The percentage difference between med on and med off scores in the preoperative period and the percentage difference between Med On/Stim On and Med off/Stim On scores in the postoperative period were accepted as levodopa response. The clinical response to DBS was calculated according to the percentage of the difference between the preoperative "on"-medication and the postoperative "medication on-stimulation on" examination scores. The doses of PD medications were adjusted at each visit and converted to the L-dopa equivalent daily dose (LEDD). Also, all patients were subjected to Hoehn Yahr, Schwab England, Beck, and Mini-Mental tests both in the preoperative and postoperative periods.

Dopamine agonists and L-dopa medications were discontinued 12 hours before the operation in all patients after tapering them down for one week and three days, respectively, before the operation. Using 1.5 Tesla and 3-Tesla MRI (Siemens, 1.5-Tesla MR Scanner, Erlangen, Germany) scanners, T2-weighted axial brain MRI with a section thickness of $2 \mathrm{~mm}$ and a single-dose contrast-enhanced T1-weighted axial brain $\mathrm{MRI}$ with a section thickness of $1 \mathrm{~mm}$ were taken in all patients 1-3 days before the operation. The dorsolateral region of the STN (motor STN) was targeted on these images at the planning station by the combination of both the indirect and direct targeting methods (Framelink-5, Medtronic, and Minneapolis, USA). Then, the tracks were determined; through which the target point was aimed. Evaluating the location of sulci and arterial structures on the track, the number of microelectrodes to be placed and that of the microelectrode recordings (MER) to be performed during the operation were noted. Following the application of stereotactic frames to all patients under local anesthesia, CT scans of the patients were taken at $1-\mathrm{mm}$ intervals in the morning of the operation. MR-CT image fusion was performed at the planning station and the coordinates were retrieved. The operations were performed under local anesthesia in the majority of the patients. The operation started in all patients contralateral to the PD-dominant side. In patients; who fail to describe a dominant side, the operation started contralateral to the side of hand dominance. A combination of intraoperative MER and macro-stimulation was used intra-operatively in all patients. In the patients operated under general anesthesia, the doses of anesthetic agents were reduced 10 minutes before the MER and possible side effects were tested by macro-stimulation. After inserting the permanent electrodes, T2-weighted brain MR or CT images at 2-mm intervals were taken to look for a possible intracranial pathology (contusion, hematoma, etc.) or check the position of the electrode. The location of the permanent electrode was confirmed through the image fusion of the postoperative MR or CT images with the preoperative MR images at the plannig station. Preceded by this imaging state; in the second stage of the surgery under general anesthesia, the permanent electrodes were connected with extension wires to a neurostimulator placed subcutaneously under the clavicle.

The statistical tests included the Wilcoxon test for the comparison of non-parametric data (preoperative/postoperative UPDRS scores), the t-test for the comparison of parametric values (preoperative-postoperative LEDD), and the linear regression analysis for testing whether the improvement in L-dopa response and UPDRS-III would predict a postoperative benefit.

\section{RESULTS}

The 49 patients included in the study were at a mean age of 
Kocabicak E. et al: Effects of DBS of the STN on the Postop L-Dopa Response

Table I: UPDRS III Scores Before and After Surgery

\begin{tabular}{ccccc}
\hline \multicolumn{2}{c}{ Preop } & \multicolumn{3}{c}{ Postop 1 year } \\
\hline Med Off & Med On & $\begin{array}{c}\text { Stim Off } \\
\text { Med Off }\end{array}$ & $\begin{array}{c}\text { Stim On } \\
\text { Med Off }\end{array}$ & $\begin{array}{c}\text { Stim On } \\
\text { Med On }\end{array}$ \\
\hline 49.1 & 20.7 & 48.7 & 19.7 & 15.1 \\
\hline
\end{tabular}

Table II: Levodopa Dosage and Response

\begin{tabular}{lccc}
\hline & Preop & Postop 1 year & Reduction \\
\hline LEDD (mg) & 1260 & 660 & $47.6 \%$ \\
\hline LED Response (\%) & 56.4 & 24.4 & $56 \%$ \\
\hline
\end{tabular}

Table III: The Other Clinical Tests

\begin{tabular}{cccccccc}
\hline $\begin{array}{c}\text { H\&Y } \\
\text { (Preop) }\end{array}$ & $\begin{array}{c}\text { H\&Y } \\
\text { (Postop) }\end{array}$ & $\begin{array}{c}\text { SCH-ENG } \\
\text { (Preop) }\end{array}$ & $\begin{array}{c}\text { SCH-ENG } \\
\text { (Postop) }\end{array}$ & $\begin{array}{c}\text { MMSE } \\
\text { (Preop) }\end{array}$ & $\begin{array}{c}\text { MMSE } \\
\text { (Postop) }\end{array}$ & $\begin{array}{c}\text { Beck } \\
\text { (Preop) }\end{array}$ & $\begin{array}{c}\text { Beck } \\
\text { (Postop) }\end{array}$ \\
\hline 3.2 & 2.3 & $44 / 100$ & $72 / 100$ & 29.2 & 28.7 & 7.9 & 7.7 \\
\hline \multicolumn{2}{c}{$\mathrm{p}<0.01$} & \multicolumn{2}{c}{$\mathrm{p}<0.01$} & \multicolumn{2}{c}{$\mathrm{p}>0.05$} & \multicolumn{2}{c}{$\mathrm{p}>0.05$} \\
\hline
\end{tabular}

Table IV: Intraoperative and Postoperative Adverse Effects

\begin{tabular}{lcc}
\hline & $\begin{array}{c}\text { Intraop } \\
\mathbf{n}(\%)\end{array}$ & $\begin{array}{c}\text { Postop } \\
\mathbf{n}(\%)\end{array}$ \\
\hline Intracranial hemorrhage & - & $1(1.9)$ \\
Seizure & - & - \\
Death & - & - \\
Local infection & - & $2(3.8)$ \\
Syncope & $2(3.8)$ & $1(1.9)$ \\
Speech disorder & $2(3.8)$ & $2(3.8)$ \\
Gait weight & - & $4(7.6)$ \\
Hypomania & - & $2(3.8)$ \\
Impulse control disorder & - & $1(1.9)$ \\
Psychosis & - & $2(3.8)$ \\
Paresthesia & $6(11.5)$ & $2(3.8)$ \\
\hline
\end{tabular}

$54.6 \pm 9$ years (from 27 to 70 years). Of these patients, 29 were men and 20 were women. $70 \%(n=34)$ of the patients had tremor dominant PD while 30\% $(n=15)$ had non-tremor dominant PD. The time elapsed from the diagnosis to surgery was 9.5 years on average and the mean follow-up period was 1.1 years. The comparison between the preoperative and postoperative scores revealed the clinical outcomes in the first year, demonstrating an improvement by $27 \%$ in the UPDRS III scores (stimulation on-medication on/ medication on). Despite the possible disease progression, the mean postoperative motor score of UPDRS III (med on/stim on) was found to be significantly lower than the preoperative score (med on) $(p<0.05)$. UPDRS III scores of the preoperative and postoperative period are presented in Table I. While preoperative LEDD was $1.260 \pm 525.1 \mathrm{mg}$, it was found to be $625.9 \pm 562.5 \mathrm{mg}$ postoperatively; showing a statistically significant reduction by $47 \%(p<0.01)$. There was not a correlation between the L-dopa responsiveness and postoperative LEDD reduction. The levodopa response significantly decreased by $56 \%$ in the first postoperative year (Table II). When the preoperative levodopa response and clinical outcomes were compared, it was observed that better clinical outcomes were achieved in the patients with higher preoperative levodopa responsiveness $(p<0.01)$. The difference between the preoperative and postoperative scores of neither MMT nor Beck depression scale were not found to be statistically significant; whereas, a significant improvement was observed in Hoehn Yahr and Schwab England scores (Table III). For MER, a total of 285 microelectrodes were implanted. The ratio of permanent electrodes inserted in the central track was $71 \%$ on the first side and $56 \%$ on the second side. In total, 97 permanent electrodes were implanted ( 49 on the first side, 48 on the second side). All patients were operated bilaterally excluding one patient. Local or general anesthesia was used for applying DBS in 46 and 3 patients, respectively. One patient developed hemorrhage which did not require surgery and did not leave permanent sequelae in the early postoperative period. No irreversible complications developed in the preoperative or postoperative period in the patients who underwent DBS (Table IV).

\section{DISCUSSION}

In this study, we evaluated the one-year data of 49 patients who were applied STN-DBS in our center and observed a strong correlation between the degree of preoperative motor responsiveness to L-dopa and the effectiveness of STN-DBS. Based on our data, the degree of L-dopa responsiveness in PD patients predicted the clinical effectiveness of STN-DBS in the first year. In the literature, there are studies that similarly showed better clinical outcomes in the early post-DBS period in patients with high preoperative levodopa responsiveness $(1,5)$. A five-year study conducted by Piboolnurak et al. found no correlation between the preoperative L-dopa responsiveness and the third and fifth-year outcomes of STN-DBS. That study argued that the preoperative L-dopa responsiveness did not predict the postoperative long-term outcomes of DBS because of the involvement of a mechanism excluding the nigrostriatal dopaminergic pathway (20). Thus; although the L-dopa responsiveness is a good indicator showing that the patient has idiopathic PD, the degree of response may not predict the outcome of STN-DBS in the long term.

Another important finding of our study is the marked decrease in levodopa responsiveness in the post-DBS period. Levodopa 
responsiveness was found to be statistically and significantly reduced by $56 \%$ in the postoperative period. Adam Zaidel et al. reported similar findings on levodopa responsiveness in their study (30). While the benefits of medication decline over time, the benefit of DBS is sustained in the motor scores in stim on/ med on states. Although levodopa responsiveness decreases with the progression of the disease, it is suggested that it cannot be explained with this situation alone $(8,15,20,21)$. Parkinson's disease is characterized by the development of rhythmic oscillatory activity ranging from 15 to $30 \mathrm{~Hz}$ in the basal ganglia. Levodopa therapy is effective in this oscillatory activity $(6,16)$. Likewise, STN-DBS acts on the pallidal and thalamic oscillator activity. Dopamine has been shown to modulate the STN-substantia nigra pars reticulata $(\mathrm{SNr})$ pathway at the presynaptic level. Electrical stimulation to STN increases the dopamine release in the $\mathrm{SNr}$ and may lead to changes in the dopamine modulation of STN-DBS in the long-term $(14,22)$. A marked decrease in this dopaminergic sensitivity, reducing the postoperative levodopa responsiveness in STN-DBS patients might result from the following factors; including a decrease in receptor sensitivity, decreased drug doses, and more direct effects of STN-DBS (19). Nevertheless, possible permanent effects of DBS during the postoperative stim-"off" period should be taken into account. In other similar studies, the difference in score between stim off-med off and stim on med on periods was considered as levedopa response. Our opinion is that when the pacemarker is closed, there may be different activities during the med off and med on periods and these results may be misleading. Therefore, when stimulation is on, med off and med on periods are compared, but it is a fact that this can mislead dopaminergic responsiveness.

In this study, a total of 285 microelectrodes were used for MER in 49 patients, and 2.9 microelectrodes on average were implanted on one side. Today, advanced magnetic resonance technologies allow for direct targeting of STN. However, MER prolongs the surgical procedure to a certain extent (12). While some studies indicated that the latter increases the $\mathrm{ICH}$ rates, there are others reporting otherwise $(3,9,27)$. The number of centers stopping MER increase day by day for this reason.

However, there is an abundance of studies in the literature, showing significant differences between the preoperative targeting and intraoperative permanent electrode localization $(11,26)$. In our study, the use of central tracks for permanent electrode localization was $71 \%$ on the first side and $56 \%$ on the second side. Intraoperative CSF loss and the error margin of stereotaxic systems have been suggested in the literature as the causes (13). Although MER is not required to determine the anatomical localization of STN, it assists the team by selecting the second most suitable track.

In this study, the majority of the patients were operated under local anesthesia, and a small number of them were operated under general anesthesia. MER was performed in all patients. While being more effective under local anesthesia, MER can be performed under general anesthesia by lowering drug doses $(7,13)$.

In the early postoperative period, one patient developed left frontal cortical hemorrhage, which did not require surgery and did not leave sequelae. Temel et al. found no differences between single and multiple microelectrode use in developing intracranial hemorrhage (25). In another two-center study of ours; 1312 microelectrodes were used in 220 patients and symptomatic intracerebral hemorrhage was observed at a rate of $1.8 \%(27)$.

Local infection developed in two (3.8\%) patients and they were treated with antibiotics. The infection did not require the DBS system to be removed. The most frequent and feared complication in DBS is the infection, and a meta-analysis on 34 studies in the literature found an infection rate of $1.6 \%$ (10). In our series, hypomania in two patients and psychosis in two patients developed in the early postoperative period. The symptoms of these patients were kept under control with psychiatric medical treatment and by altering the stimulation settings. One of the most frequent postoperative complications occurring after STN-DBS is early hypomania and psychosis. Hence, vigilance is required in the early postoperative period and patients should be followed up closely.

\section{CONCLUSION}

The responsiveness to L-dopa in our study decreased after applying STN-DBS; however, the reasons for this finding have not been clarified. However, STN-DBS allows for the reduction in the doses of PD drugs and improves the activities of daily living, motor function, motor fluctuations, and dyskinesia. While the benefits of medication decline over time, the benefits of DBS in motor scores in stim "on"/med "on" states sustain significantly in the first year.

\section{REFERENCES}

1. Benabid AL, Chabardes S, Mitrofanis J, Pollak P: Deep brain stimulation of the subthalamic nucleus for the treatment of Parkinson's disease. The Lancet Neurology 8(1):67-81, 2009

2. Benabid AL, Pollak P, Gross C, Hoffmann D, Benazzouz A, Gao DM, Laurent A, Gentil M, Perret J: Acute and long-term effects of subthalamic nucleus stimulation in Parkinson's disease. Stereotact Funct Neurosurg 62:76-84, 1994

3. Ben-Haim S, Asaad WF, Gale JT, Eskandar EN: Risk factors for hemorrhage during microelectrode-guided deep brain stimulation and the introduction of an improved microelectrode design. Neurosurgery 64(4):754-763, 2009

4. Camlidag I, Kocabicak E, Sahin B, Jahanshahi A, Incesu L, Aygun D, Yildiz O, Temel Y, Belet U: Volumetric analysis of the subthalamic and red nuclei based on magnetic resonance imaging in patients with Parkinson's disease. Int J Neurosci 124:291-295, 2014

5. Charles PD, Van Blercom N, Krack P, Lee SL, Xie J, Besson G, Benabid AL, Pollak P: Predictors of effective bilateral subthalamic nucleus stimulation for PD. Neurology 59:932934, 2002

6. Doyle LMF, Kühn AA, Hariz M, Kupsch A, Schneider GH, Brown, P: Levodopa-induced modulation of subthalamic beta oscillations during self-paced movements in patients with Parkinson's disease. European Journal of Neuroscience 21(5): 1403-1412, 2005 
7. Harrie AM, Kausar J, Roberts SA, Mocroft AP, Hodson JA, Pall HS, Mitchell R: Deep brain stimulation of the subthalamic nucleus for advanced Parkinson disease using general anesthesia: Long-term results. Journal of Neurosurgery 116(1): 107-113, 2012

8. Hilker R, Portman AT, Voges J, Staal MJ, Burghaus L, Van Laar T, Herholz K: Disease progression continues in patients with advanced Parkinson's disease and effective subthalamic nucleus stimulation. J Neurol Neurosurg Psychiatry 76(9):1217-1221, 2005

9. Kimmelman J, Duckworth K, Ramsay T, Voss T, Ravina B, Emborg ME: Risk of surgical delivery to deep nuclei: A metaanalysis. Movement Disorders 26(8):1415-1421, 2011

10. Kleiner-Fisman G, Herzog J, Fisman DN, Tamma F, Lyons KE, Pahwa R, Deuschl G: Subthalamic nucleus deep brain stimulation: Summary and meta-analysis of outcomes. Movement disorders: Official journal of the Movement Disorder Society 21(S14):S290-S304, 2006

11. Kocabicak E, Alptekin O, Ackermans L, Kubben P, Kuijf M, Kurt E, Esselink R, Temel Y: Is there still need for microelectrode recording now the subthalamic nucleus can be well visualized with high field and ultrahigh MR imaging. Front Integr Neurosci 9:46, 2015

12. Kocabicak E, Alptekin O, Aygun D, Yildiz O, Temel Y: Microelectrode recording for deep brain stimulation of the subthalamic nucleus in patients with advanced parkinson's disease: Advantage or loss of time? Turkish Neurosurgery 29(5): 677-682, 2019

13. Kocabicak E, Aygun D, Alptekin O, Guz H, Kurt M, Sarihasan B, Temel, Y: Conversion of local anesthesia-guided deep brain stimulation of the subthalamic nucleus to general anesthesia. J Neurol Surg A Cent Eur Neurosurg 74(5):332334, 2013

14. Kocabicak E, Jahanshahi A, Schonfeld L, Hescham SA, Temel Y, Tan S: Deep brain stimulation of the rat subthalamic nucleus induced inhibition of median raphe serotonergic and dopaminergic neurotransmission. Turk Neurosurg 25(5):721727, 2015

15. Krack P, Batir A, Van Blercom N, Chabardes S, Fraix V, Ardouin C, Benabid AL: Five-year follow-up of bilateral stimulation of the subthalamic nucleus in advanced Parkinson's disease. New England Journal of Medicine 349(20):19251934, 2003

16. Kühn AA, Kupsch $A$, Schneider GH, Brown P: Reduction in subthalamic 8-35 Hz oscillatory activity correlates with clinical improvement in Parkinson's disease. European Journal of Neuroscience, 23(7), 1956-1960, 2006

17. Kumar R, Lozano AM, Kim YJ, Hutchison WD, Sime E. Halket E, Lang AE: Double-blind evaluation of subthalamic nucleus deep brain stimulation in advanced Parkinson's disease. Neurology 51(3):850-855, 1998
18. Morishita T, Rahman M, Foote KD, Fargen KM, Jacobson 4th CE, Fernandez HH, Rodriguez RL, Malaty IA, Bowers D, Hass CJ, Katayama Y, Yamamoto T, Okun MS: DBS candidates that fall short on a levodopa challenge test: Alternative and important indications. Neurologist 17(5):263-268, 2011

19. Moro E, Esselink RJ, Benabid AL, Pollak P: Response to levodopa in parkinsonian patients with bilateral subthalamic nucleus stimulation. Brain 125(11):2408-2417, 2002

20. Piboolnurak P, Lang AE, Lozano AM, Miyasaki JM, SaintCyr JA, Poon YYW, Moro E: Levodopa response in longterm bilateral subthalamic stimulation for Parkinson's disease. Movement Disorders 22(7):990-997, 2007

21. Rodriguez-Oroz MC, Zamarbide I, Guridi J, Palmero MR, Obeso JA: Efficacy of deep brain stimulation of the subthalamic nucleus in Parkinson's disease 4 years after surgery: Double blind and open label evaluation. J Neurol Neurosurg Psychiatry 75(10):1382-1385, 2004

22. Rosales MG, Flores G, Hernández S, Martínez-Fong D, Aceves J: Activation of subthalamic neurons produces NMDA receptor-mediated dendritic dopamine release in substantia nigra pars reticulata: $A$ microdialysis study in the rat. Brain Research 645(1-2):335-337, 1994

23. Schrag A, Quinn N: Dyskinesias and motor fluctuations in Parkinson's disease: A community-based study. Brain 123(11): 2297-2305, 2010

24. Schuepbach WMM, Rau J, Knudsen K, Volkmann J, Krack P, Timmermann L, Falk D: Neurostimulation for Parkinson's disease with early motor complications. New England Journal of Medicine 368(7):610-622, 2013

25. Temel Y, Wilbrink P, Duits A, Boon P, Tromp S, Ackermans L, Visser-Vandewalle V: Single electrode and multiple electrode guided electrical stimulation of the subthalamic nucleus in advanced Parkinson's disease. Operative Neurosurgery 61 Suppl 5:ONS346-ONS357, 2007

26. Toda H, Sawamoto N, Hanakawa T, Saiki H, Matsumoto S, Okumura R, Hashimoto N: A novel composite targeting method using high-field magnetic resonance imaging for subthalamic nucleus deep brain stimulation. Journal of Neurosurgery 111(4):737-745, 2009

27. Tonge M, Ackermans L, Kocabicak E, van Kranen-Mastenbroek V, Kuijf M, Oosterloo M, Kubben P, Temel Y: A detailed analysis of intracerebral hemorrhages in DBS surgeries. Clin Neurol Neurosurg 139:183-187, 2015

28. Vingerhoets FJ, Villemure JG, Temperli P, Pollo C, Pralong E, Ghika J: Subthalamic DBS replaces levodopa in Parkinson's disease: Two-year follow-up. Neurology 58(3):396-401, 2002

29. Yamada K, Goto S, Hamasaki T, Kuratsu Jl: Effect of bilateral subthalamic nucleus stimulation on levodopaunresponsive axial symptoms in Parkinson's disease. Acta Neurochirurgica 150(1):15-22, 2008

30. Zaidel A, Bergman H, Ritov YA: Levodopa and subthalamic deep brain stimulation responses are not congruent. Movement Disorders 25(14):2379-2386, 2010 